Archives

$43 \mid 2009$

Hommage à Jacques Ozouf

\title{
Bibliographie de Jacques Ozouf
}

\section{Mayyada Kheir}

\section{OpenEdition}

\section{Journals}

Édition électronique

URL : http://journals.openedition.org/ccrh/3515

DOI : $10.4000 /$ ccrh.3515

ISSN : $1760-7906$

Éditeur

Centre de recherches historiques - EHESS

Édition imprimée

Date de publication : 25 janvier 2009

Pagination : 133-138

ISSN : 0990-9141

Référence électronique

Mayyada Kheir, «Bibliographie de Jacques Ozouf », Les Cahiers du Centre de Recherches Historiques [En ligne], 43 | 2009, mis en ligne le 17 février 2012, consulté le 08 mai 2019. URL : http://

journals.openedition.org/ccrh/3515; DOI : 10.4000/ccrh.3515

Ce document a été généré automatiquement le 8 mai 2019.

Article L.111-1 du Code de la propriété intellectuelle. 


\title{
Bibliographie de Jacques Ozouf
}

\author{
Mayyada Kheir
}

\section{Ouvrages}

1958

1 Jacques Ozouf et Firmin Lentacker, Jean-Paul Moreau, Yves Pasquier, Nouveau cours de géographie, classes terminales des lycées et collèges, Paris, F. Nathan, 1958, 544 pages.

1967

2 Nous les maîtres d'école. Autobiographies d'instituteurs de la Belle Époque, présentées par Jacques Ozouf, Paris, Julliard [coll. « Archives », n²7], 1967, 272 pages.

3 - Rééditions :

4 Gallimard/Julliard [coll. « Archives », n²7], 1973, 272 pages [ré-impression en 1983].

5 Gallimard [coll. « Folio Histoire », n 50], 1993, 312 pages [ré-impression 1999].

1970

6 Vincent Auriol, Mon septennat: 1947-1954, notes de journal présentées par Pierre Nora et Jacques Ozouf, Paris, Gallimard [coll. « Témoins »], 1970, VIII-609 pages [cet ouvrage est constitué d'extraits du Journal, dont les sept volumes seront publiés de 1970 à 2003 ; voir plus bas].

7 Vincent Auriol, Journal du septennat : 1947-1954, sous la direction de Pierre Nora et Jacques Ozouf. Tome I, 1947, version intégrale établie, introduite et annotée par Pierre Nora, préface de René Rémond, Paris, A. Colin, 1970, LXX-878 pages.

1971

8 Vincent Auriol, Journal du septennat : 1947-1954, sous la direction de Pierre Nora et Jacques Ozouf. Tome VII, 1953-1954 ; version intégrale établie, introduite et annotée par Jacques Ozouf, Paris, A. Colin, 1971, XLVI-863 pages.

1974 
Ozouf. Tome II, 1948, version intégrale établie par Edmond Mouret et Jean-Pierre Azéma ; introduite et annotée par Jean-Pierre Azéma, Paris, A. Colin, 1974, XXXI-739 pages.

\section{5}

10

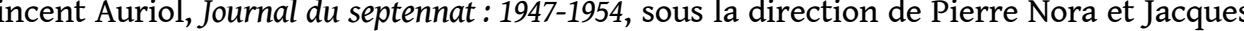
Ozouf. Tome V, 1951 ; version intégrale établie, introduite et annotée par Laurent Theis, Paris, A. Colin, 1975, XX-771 pages.

1977

11 Vincent Auriol, Journal du septennat : 1947-1954, sous la direction de Pierre Nora et Jacques Ozouf Tome III, 1949, version intégrale, établie, introduite et annotée par Pierre Kerleroux, Paris, A. Colin, 1977, XXVII-657 pages.

12 Jacques Ozouf et François Furet, Lire et écrire : l'alphabétisation des Français de Calvin à Jules Ferry; [recherche conduite dans le cadre d'un séminaire du Centre de recherches historiques de l'École des hautes études en sciences sociales de 1972 à 1975], Paris, Éditions de Minuit [coll. «le sens commun »], ouvrage publié avec le concours du CNRS, 2 vol. , 1977: vol. 1, bilan général de l'enquête, rédigé par François Furet et Jacques Ozouf, 390 pages.

vol. 2, études régionales, rédigées par M. Jeorger, V. Nahoum, M.L. Netter, Y. Pasquet, M.M. Compère, P. Butel, G. Mandon, J.P. Poussou et P. Lévêque, 378 pages.

15 - Réédition à l'identique en 1991.

16 - Traduction: Reading and writing: literacy in France from Calvin to Jules Ferry, Cambridge/ London/N.Y., Éditions de la MSH/Cambridge University Press [coll. « Cambridge Studies in oral and literate Culture », vol. 5], 1983, 369 pages. [Seul le premier des deux volumes de l'édition française est ici traduit.]

1978

17 Vincent Auriol, Journal du septennat : 1947-1954, sous la direction de Pierre Nora et Jacques Ozouf. Tome VI, 1952 ; version intégrale établie, introduite et annotée par Dominique Boché, Paris, A. Colin, 1978, LI-1227 pages.

1992

18 Jacques Ozouf et Mona Ozouf, La République des instituteurs, avec Véronique Aubert et Claire Steindecker, Paris, Gallimard / le Seuil [coll. « Hautes Etudes »], 1992, 386 pages.

19 - Réédition: Paris, Gallimard / le Seuil [coll. « Points Histoire »], 2000, 487 pages.

\section{3}

20 Vincent Auriol, Journal du septennat: 1947-1954, sous la direction de Pierre Nora et Jacques Ozouf. Tome IV, 1950 [avec un CD-ROM réunissant les sept tomes], version intégrale établie, introduite et annotée par Anne-Marie Bellec, avec la collaboration de Violaine Chatelain, avant-propos de Pierre Nora, Paris, Tallandier, 2003, 799 pages.

\section{Articles, recensions}

1963

21 «L'enquête d'opinion en histoire. Un exemple: l'instituteur français, 1900-1914 ", Le Mouvement social, n 44, juil.-sept. 1963, p. 3-22. 
1964

22 Jacques Ozouf et Mona Ozouf, "Le thème du patriotisme dans les manuels primaires ", Le Mouvement social, n 49, oct.-déc. 1964, p. 5-31.

1965

23 «Études de presse et analyse du contenu », Le Mouvement social, n 53, oct.-déc. 1965, p. 39-49.

1966

24 «L'Humanité et les journées de février 1934 », Le Mouvement social, n 54, janv.-mars 1966, p. 151-171.

25 "Les instituteurs de la Manche et leurs associations au début du XX siècle », Revue d'histoire moderne et contemporaine, vol. 13, n 1, janv.-mars 1966, p. 95-114.

26 «Mesure et démesure: l'étude de l'opinion", Annales: économies, sociétés, civilisations, vol. 21, n² 2, 1966, p. 324-345.

27 Jacques Ozouf et François Furet, «La France a-t-elle changé ? », Preuves, n 180, fév. 1966, p. 47-55.

1967

28 "Les instituteurs qui ont fait la République » [interview au sujet de Nous les maîtres d'école ], Réalités, n² 253, février 1967, p. 99-103.

1969

29 «Paul Gerbod, La Condition universitaire en France au XIX ${ }^{e}$ siècle » [compte rendu], Annales: économies, sociétés, civilisations, vol. 24, n² 2, 1969, p. 512-516.

1971

«J. P. Azéma et M. Winock, La IIIe République » [compte rendu], Annales : économies, sociétés, civilisations, vol. 26, $\mathrm{n}^{\circ}$ 5, 1971, p. 1085-1087.

31 «Les élections législatives de mars 1967, Cahiers de la Fondation nationale des sciences politiques » [compte rendu], Annales : économies, sociétés, civilisations, vol. 26, $\mathrm{n}^{\circ}$ 5, 1971, p. 1090-1091.

1973

« Géographie des élections législatives de mars 1973 », Esprit, juin 1973, p. 1295-1346.

1974

33 « L'élection présidentielle de mai », Esprit, juil.-août 1974, p. 14-37.

$34 \quad 1975$

35 «Le PCF » [table ronde, avec J.M. Domenach, G. Lavau et M. Winock], Esprit, fév. 1975, p. $173-200$.

1976

36 Jacques Ozouf et François Furet, «Literacy and industrialization: the case of the département du Nord in France ", Journal of European economic History, vol. 5, n 1, 1976, p. 5-44.

1977

37 Jacques Ozouf et François Furet, «L'alphabétisation : trois siècles de métissage culturel ", Annales : économies, sociétés, civilisations, vol. 32, n 3, 1977, p. 488-502. 

1976 siècle ", in Mélanges d'histoire sociale offerts à Jean Maitron, Paris, Éd. de l'atelier/Éd. ouvrières, 1976, p. 167-176.

1977

44 statistique, Journées d'étude de l'INSEE sur l'histoire de la statistique, tome I: Contributions, Vaucresson, 23, 24 et 25 juin 1976, INSEE, 1977, p. 139-153.

Réédition [adjoint d'un second volume, auquel J. Ozouf n'a pas participé]: INSEE / Economica, 1987.

1981

«Retour sur une enquête", in Cent ans d'école, par le groupe de travail de la maison d'école de Montceau-les-Mines, Seyssel, Éditions du Champ Vallon [coll. «Milieux»], 1981, p. 25-35.

47 Jacques Ozouf et François Furet, «Deux légitimations historiques de la société française au XVII e siècle : Mably et Boulainvilliers ", in Béla Köpeczi \& Eva H. Balázs (éd.), Noblesse française, noblesse hongroise, $\mathrm{XVI}^{e}$-XIX ${ }^{e}$ siècles, Actes du colloque franco-hongrois d'histoire sociale, Rennes, 11, 12 et 13 juin 1975, Budapest/Paris, Akadémiai Kindó/Ed. du CNRS, 1981, p. 65-76.

48 Jacques Ozouf et François Furet, "Three centuries of cultural cross-fertilization: France », in Harvey J. Graff (éd.), Literacy and social Development in the West: a reader, Londres/New York/Malbourne, Cambridge Univesity Press [coll. « Cambridge Studies in oral and literate Culture ", $n^{\circ}$ 5], 1981, p. 214-239. [Reproduction de la conclusion de Reading and Writing, avec permission de l'éditeur.]

1984

49 Jacques Ozouf et Mona Ozouf, « Le tour de France par deux enfants », in Pierre Nora (éd.), Les lieux de mémoire, première partie : la République. Paris, Gallimard [coll. « NRF »], 1984, p. 291-321.

50 - Traduction: «Le tour de la France par deux enfants: The Little Red Book of the Republic », in Pierre Nora (éd.), et Lawrence D. Kritzman (éd. pour l'édition anglaise), Realms of Memory, vol. 2 : Traditions, N.Y., Columbia University Press, p. 125-150.

51 - Réédition : tome I, Paris, Gallimard [collection « Quarto »], 1997, p. 277-301. 
1985

52 Jacques Ozouf et Mona Ozouf, «Préface» in La Forteresse enseignante: la Fédération de l'Éducation nationale, par Véronique Aubert, Alain Bergounioux, Jean-Paul Martin, René Mouriaux, Paris, Fayard, 1985, p. I-XIII.

1988

Jacques Ozouf et Mona Ozouf, «Préface » in Lavisse (Ernest), Souvenirs, Paris, CalmannLévy, 1988, XVIII-287 p. VII-XVIII.

54 Direction de thèses

55 Béatrice Çakirogĺu, «Les Monarchistes révolutionnaires : recherche sur l'aile gauche de l'Action Française, 1906-1914 », EHESS, 1977.

56 Yolande Cohen, «Les mouvements de jeunesse socialiste en France : espoirs et échecs (1880-1905) », EHESS, 1978.

\section{AUTEUR}

MAYYADA KHEIR

EHESS/CEIFR 